\title{
Reliability of Component based Software System using Soft Computing Techniques - A Review
}

\author{
Jyoti Agarwal \\ Mtech CSE \\ Amity University, Noida
}

\author{
Renuka Nagpal \\ Assistant Professor \\ Amity University, Noida
}

\author{
Rajni Sehgal \\ Assistant Professor \\ Amity University, Noida
}

\begin{abstract}
Component Based Software Engineering is based on reusability of code. It is an approach which let customer to have quality product by paying less amount of money and spending less time to produce. In this paper we will find out the factors which greatly effects reliability of component based system by conducting literature survey. The focus of this paper is to provide an overview of estimation of reliability factors from literature survey. We will prioritized reliability factors by applying soft computing techniques which in turn result factor which greatly effects reliability of component based system.
\end{abstract}

\section{Keywords}

Reliability, Component based Software Engineering, Soft computing techniques

\section{INTRODUCTION}

In the field of Software Engineering Component based Software is a recent approach that emphasize on design and development of component based software system. It has benefits to software design, implementation, and maintenance and architecture flexibility. Also ensuring reliability of a component based software system is important for its successful application in safety and mission critical systems. Reliability is defined as the probability that no failures occur for a specific period of time under a set of specific environment conditions. There are many factors which effects reliability of a component based system. In this paper we will find out factors which greatly effects reliability of component based system and which we will prioritized by applying soft computing techniques

This paper is organized as follows: Section 2 describes Component based software engineering, Section 3 defines reliability, Section 4 describes soft computing techniques, Section 5 presented literature survey, Section 6 describes proposed work, Section 7 describes research methodology, Section 8 summarizes the paper and Section 9 presented future work.

\section{COMPONENT BASED SOFTWARE ENGINEERING}

Component-Based Software Engineering (CBSE) is a technology uses reusable software component to develop a complex system which satisfy user requirement. It is based on reusable software component that can be replaced and updated easily without affecting the software equality. So CBSE play an important role to enhance the quality through reusability to build component-based software from the pre-fabricated reusable and testable component .CBSE is very helpful to improve the productivity. But to maintain the quality it is necessary to measure the attributes of the software to make it reliable and reusable. In CBSE, to make reliable and reusable software it is very necessary to check the quality at every phase before testing to detect the faults at early stage of software development lifecycle.

\section{RELIABILITY}

The most important characteristics of software is reliability. Thus, reliability can be defined as the quality of the fault free delivery of service for a particular period of time within the specified constraints. As software becomes more and more crucial to the operation of the systems on which we depend, the software should behave in the way it is intended, or it should meet the requirements for which is defined and designed .Ensuring high level of reliability for the software system assures the overall operational success of the system. Software reliability is defined as the probability of the failurefree operation of a software system for a specified period of time in a specified environment [22].

\section{SOFT COMPUTING TECHNIQUES}

In soft computing the problem is represented in such a way that the state of the system can somehow be calculated and compared to some desired state. It involves methods which operate in an environment which subject to uncertainty and imprecision. The main components of Soft Computing are Fuzzy Logic, Probabilistic Reasoning, Neural Computing and Genetic Algorithms. Fuzzy logic is concerned with imprecision, approximate reasoning and representation of aspects that are only qualitatively known; Probabilistic Reasoning such as Bayesian Belief Networks has main characteristic is its ability to update previous outcome estimates by conditioning them with newly available evidence ; Neural Computing focuses on the understanding of neural networks and learning systems, self-organizing structures, and the implementation of models from available data ;Genetic and Evolutionary Computing provide approaches to computing based on analogues of natural selection, such as, the optimization methods based on ant colonies or on particle swarms.

\section{LITERATURE SURVEY}

At this section we make a brief review of reliability of component based software systems in ascending order depending on the year of publication and summarized reliability factors in tabular form.

\subsection{In 1999, William W. Everett[1]}

He described an approach to analyze software reliability using component analysis which allows us to do reliability analysis earlier in the life cycle. He described how to estimate model parameters from the characteristics of the software 
components and characteristics of how test cases and operational usage stress the software components.

\subsubsection{Main contribution}

He described six steps for doing software component reliability analysis which are:

1) Divide the software into components

2) Characterize the properties of each component

3) Characterize how usage stresses each component

4) Model reliability growth of each component

5) Superimpose component reliabilities

6) Do "confirmatory" analysis during testing

He also did comparison of his approach to other approach that were used to analyze software reliability growth during testing

\subsection{In 2000, Martin Kappes, Reinhard P.K lemm and Chandra M. R. Kintala[2]}

They presented model to analyze reliability of component based software system .They modeled component as a finite state machine with an associated reliability figure expresses overall reliability of the component. They proved by their model that no algorithm can accurately calculate the reliability of a system of finite state machine with known or estimated overall reliabilities or transition failure probabilities.

\subsubsection{Main contribution}

They have showed that there is no single algoritm to compute either precisely or approximately the reliability of an arbitrary software system given the reliabilities of the components in that system or the reliabilities of operations in the components. Their findings did not imply that reliability of a specific software system cannot be determined but it implied that every method that attempts to compute or approximate the overall software system reliability from given operation or component reliabilities there will be software systems for which this method fails.

\subsection{In 2001, Dick Hamlet, Dave Mason and Denise Woit [3]}

They described how component developers can design and test their components to produce measured that is later used by system designers to calculate composite system reliability without implementation and test of system being design.

\subsubsection{Main contribution}

Their theory described how to make component measurements that are independent of operational profiles and how to incorporate the overall software system level operational profile into the system reliability calculations. They addressed basic technical problem inherent in certifying components to be released for later use in an arbitrary system.

\subsection{In 2001, Hongxia Jin and P.Santhanam}

[4]

They argued that in order to achieve high reliability of software system built from existing components, we need to use reliable component that can interact properly.

\subsubsection{Main contribution}

They presented unified approach to do an effective evaluation. They presented way to describe component at a semantic level. They presented systematic way to capture arbitrary component interaction mechanism using multidimensional space. Their approach detected mismatch between interacting components in the new system before they are actually integrated into the system. They guided how to design the adaption mechanism to bridges the mismatches.

\subsection{In 2002, Jung-Hua Lo,Sy-Yen \\ Kuo,Michael R.Lyu and Chin-Yu Huang[5]}

They have proposed an analytical approach for estimating reliability of component based software system. They assumed that software components are heterogeneous and transfer of control between components follow a discrete time markov process

\subsubsection{Main contribution}

They presented new approach to analyze reliability of component based software system based on the reliabilities of the individual components and architecture of the system. They also derived some useful mathematical properties to show that model is very powerful. They have showed experimentally that proposed methods can solve the testing effort allocation problems and improve quality and reliability of the software system.

\subsection{In 2003, Mao Xiaoguang and Deng Yongjin [6]}

They presented a general model component probability transition diagram which is compatible with different kinds of components and enable reliability tracing through component based software process.

\subsubsection{Main contribution}

They have constructed a reliability tracing system consisting 4 parts: modeling component, reliability analyzer, reliability specifying component and version controller.

\subsection{In 2004, Sherif Yacoub , Bojan Cukic and Hany H.Ammar [7]}

They have introduced a reliability model and reliability analysis technique for component based software .The technique were named scenario based reliability analysis (SBRA).

\subsubsection{Main contribution}

They proposed SBRA which is a stack based algorithm suitable for analyzing sensitivity of system level reliability as a function of component link and subsystem reliabilities. Algorithm application results in identifying critical components, subsystems and links which required increase attention in testing, verification and validation

\subsection{In 2006, Yoshinobu Tamura and Shigeru Yamada [9]}

They have focused on the open source software such as Calendar, Thunderbird and Xen and discussed their method of reliability assessment. They have applied especially neural network in order to consider the effect of each software component on the reliability of an entire system under such open source development paradigm. 


\subsubsection{Main contribution}

By using neural network they have proposed method of reliability assessment incorporating the interaction among software components. The neural network and NHPP model applied which have simple structure therefore they can easily apply their method to actual open source software by role.

\subsection{In 2008, WANG Dong, HUANG Ning and YE Ming [10]}

They analyzed various complex component relationships (parallel, loops, backup, fault tolerance, request and response, etc.)How to influence the reliability of the whole system and solved these complicated relationship to satisfy the Markov property

\subsubsection{Main contribution}

They extends the scope of application of Markov property with the assumption that reliabilities of all components and transition probabilities are given but in early stage of the software life cycle this information is difficult to obtain .They analyzed complex relationship among components which has extended scope to improve accuracy of reliability estimation

\subsection{In 2008, Fan Zhang, Xing she Zhou, Junwen Chen and Yunwei Dong [11]}

They introduced a sub domain based analysis approach to characterize the component into path based architecture reliability model and provide the enhanced composition algorithms to solve the model

\subsubsection{Main contribution}

They believed path based architecture reliability model fully capture the effect of different operational profile on the overall reliability of system from aspects of both transition and component reliability. This has made reliability analysis based on architecture more accurate and flexible.

\subsection{In 2009, Francesa Saqlietti,Florino Pinte and Sven Sohnlein [12]}

Their paper presented extensive integration testing phases recommended for the purpose of exposing interaction faults.

\subsubsection{Main contribution}

They have propose two approaches improving state-of-the-art concerning verification and validation of complex component based software. Extensive systematic integration testing is supported by an automatic generator of test cases aimed at achieving high interaction coverage by a low number of test. Determination of system reliability estimates at sharp confidence levels is achieved by statistical sampling theory making use of testing operational data gained with pre developed components.

Both approaches were succeed in reducing the prohibitive effort required by rigorous verification techniques and contribute practicability in real world environments.

\subsection{In 2010, Ate F Mohamed and Mohammad Zulkernine [13]}

They proposed failure type aware reliability quantification of fault tolerant component based software systems.

\subsubsection{Main contribution}

They incorporated component failure dependency in reliability quantification by analyzing failure propagation among system components. They have analyzed failure occurrence, permeation, unmasking and propagation in the fault tolerant components and the architectural service routes among them. They aggregate impact of these failures on system reliability .

\subsection{In 2011, Deepak Panwar and Pradeep Tomar [14]}

In their paper they have calculated maximum number of faults through investigating the reliability and reusability of component based software. Halsted software science was used which is very effective to minimize the cost and time for a component based system. This method find effects on reusability through changes in initial phases of software developing process like requirement analysis,design,coding and simultaneously on reliability of code or program which would be helpful to find number of faults.

\subsubsection{Main contribution}

They focused on reliability and reusability to make quality software. They summarized affects on software reliability and reusability due to the changes in the software requirement, design, code, component complexity, software complexity .They gave general factors which affect the reliability and reusability calculate two variables reliability and reusability .

\subsection{In 2012, Aditya Pratap Singh and Pradeep Tomar [15]}

They proposed a Reliability estimation model for a component based system to estimate the reliability through path propagation probability and component impact factor.

\subsubsection{Main contribution}

The Reliability estimation model they proposed is suitable for system whose design is based on valid scenarios and activity diagrams. Their proposed model is useful to estimate the reliability of component based system and can be used adaptively in early stages of software development.

Reliability factors which we got from literature survey are shown in table below: 
Table 1. Reliability factors of component based Software System

\begin{tabular}{|c|c|c|}
\hline Source & Purpose & $\begin{array}{l}\text { Reliability } \\
\text { Factors }\end{array}$ \\
\hline $\begin{array}{c}\text { William W. } \\
\text { Everett, Software } \\
\text { Component } \\
\text { ReliabilityAnalysis } \\
\text {,IEEE,1999 }\end{array}$ & $\begin{array}{l}\text { to estimate model } \\
\text { parameters from the } \\
\text { characteristics of the } \\
\text { software } \\
\text { components }\end{array}$ & $\begin{array}{c}\text { Design tradeoff } \\
\text {,operational stress }\end{array}$ \\
\hline $\begin{array}{c}\text { Dick Hamlet, Dave } \\
\text { Mason, Denise } \\
\text { Woit, Theory of } \\
\text { Software } \\
\text { Reliability Based } \\
\text { on Components, } \\
\text { IEEE,2001 }\end{array}$ & $\begin{array}{l}\text { foundational theory } \\
\text { of software system } \\
\text { reliability based on } \\
\text { components }\end{array}$ & $\begin{array}{c}\text { Operational } \\
\text { profile ,Spike in } \\
\text { intermediate } \\
\text { profile }\end{array}$ \\
\hline $\begin{array}{c}\text { Hongxi Jin } \\
\text { „P.Santhanam ,An } \\
\text { Approach to } \\
\text { Higher Reliability } \\
\text { Using Software } \\
\text { Components } \\
\text {,IEEE,2001 }\end{array}$ & $\begin{array}{c}\text { Detected mismatch } \\
\text { between interacting } \\
\text { components in the } \\
\text { new system before } \\
\text { they are actually } \\
\text { integrated into the } \\
\text { system }\end{array}$ & Interoperability \\
\hline $\begin{array}{c}\text { H. Singh, V. } \\
\text { Cortellessa, B. } \\
\text { Cukic, E. Gunel, } \\
\text { V. Bharadwaj, A } \\
\text { bayesian approach } \\
\text { to reliability } \\
\text { prediction and } \\
\text { assessment of } \\
\text { component based } \\
\text { systems,IEEE,2001 }\end{array}$ & $\begin{array}{l}\text { Bayesian estimation } \\
\text { framework, } \\
\text { posterior probability } \\
\text { of failure is } \\
\text { calculated from the } \\
\text { priors and test } \\
\text { failure data }\end{array}$ & $\begin{array}{l}\text { usage profile } \\
\text {,exception } \\
\text { handling,fault } \\
\text { tolerance } \\
\text { mechanism } \\
\text {,system } \\
\text { configuration }\end{array}$ \\
\hline $\begin{array}{l}\text { Francesca Saglietti, } \\
\text { Florin Pinte, Sven } \\
\text { Söhnlein, } \\
\text { Integration and } \\
\text { reliability testing } \\
\text { for component- } \\
\text { based software } \\
\text { systems, 35th } \\
\text { euromicro } \\
\text { conference on } \\
\text { software } \\
\text { engineering and } \\
\text { advanced } \\
\text { applications, } 2009 .\end{array}$ & $\begin{array}{l}\text { proposed two } \\
\text { approaches } \\
\text { improving state-of- } \\
\text { the-art concerning } \\
\text { verification and } \\
\text { validation of } \\
\text { complex component } \\
\text { based software }\end{array}$ & $\begin{array}{c}\text { Application } \\
\text { context }\end{array}$ \\
\hline
\end{tabular}

\begin{tabular}{|c|c|c|}
\hline $\begin{array}{l}\text { Deepak panwar, } \\
\text { Pradeep Tomar, } \\
\text { New method to } \\
\text { find the maximum } \\
\text { number of faults by } \\
\text { analyzing } \\
\text { reliability and } \\
\text { reusability in } \\
\text { component-based } \\
\text { software, IEEE } \\
\text {,2011 }\end{array}$ & $\begin{array}{c}\text { focused on } \\
\text { reliability and } \\
\text { reusability to make } \\
\text { quality software }\end{array}$ & $\begin{array}{l}\text { cohesion } \\
\text {,coupling } \\
\text {,software } \\
\text { complexity } \\
\text {,component } \\
\text { complexity }\end{array}$ \\
\hline $\begin{array}{c}\text { Pankaj Jalote, } \\
\text { Brendan Murphy, } \\
\text { Mario Garzia, Ben } \\
\text { Errez,Measuring } \\
\text { Reliability of } \\
\text { Software Products } \\
\text {,Microsoft } \\
\text { Corporation,2003 }\end{array}$ & $\begin{array}{l}\text { Discussed key } \\
\text { issues in } \\
\text { determining } \\
\text { reliability of } \\
\text { software products }\end{array}$ & Usage time \\
\hline
\end{tabular}

\section{PROPOSED WORK}

From literature survey we can say that no previous work has done to prioritized reliability factors which affect component based software system. So we will find out factors which greatly effects reliability of component based software system and then consider three different version of windows operating system (Windows 7 , Windows 8 , windows 8.1 ) as case study and propose which version of windows operating system is most reliable on the basis of considered factors.

\section{RESEARCH METHODOLOGY}

First of all conduct theoretical analysis for research problem from literature survey and find out factors on which reliability of component based software system depends. Then consider three different version of windows operating system as case study and select few reliability factors according to case study. Then prepare questionnaire and conduct survey to get user view on effect of reliability factor on different version of windows operating system. Analyze survey result and prepare weight matrix and apply soft computing technique like fuzzy TOPSIS, fuzzy AHP which give ranking to the different alternatives on the basis of criteria we considered and get most reliable version of windows operating system .Also we will compare priority obtained by fuzzy AHP and fuzzy TOPSIS which result in appropriate method to find out the most reliable version of windows operating system.

\section{CONCLUSION}

This paper presented literature review on reliability of component based software system .This paper focuses on reliability factors of a component based software system which has been found after conducting theoretical survey. Case study considered in this paper is different windows operating system versions which are Windows 7, Windows 8, and Windows 8.1. Research methodology has defined to find out most reliable windows operating system.

\section{FUTURE WORK}

There is further scope of improvement in reliability of component based software system by find out sub factors which affect reliability of component based software system. 
As proposed work will give most reliable version of windows operating system which will help users to work on most reliable platform .It will help developer to design component based software system by considering reliability factors on which every component based software system depends. There is scope to find out most reliable android version and help user to choose best version Smartphone out of different version available.

\section{REFERENCES}

[1] William W. Everett, Software Component Reliability Analysis, IEEE, 1999.

[2] Martin Kappes , Reinhard P.K lemm , Chandra M.R.Kintala ,Formal Limits on Determining Reliabilities of Component-Based Software Systems,IEEE,2000.

[3] Dick Hamlet, Dave Mason , Denise Woit , Theory of Software Reliability Based on Components , IEEE,2001.

[4] HONGXIA JIN , P.Santhanam, An Approach to Higher Reliability Using Software Components ,IEEE,2001.

[5] Jung-Hua Lo,Sy-Yen Kuo,Michael R.Lyu , Chin-Yu Huang, Optimal Resource Allocation and Reliability Analysis for Component-Based Software Applications, Proceedings of the 26 th Annual International Computer Software and Applications Conference (COMPSAC'02) ,2002.

[6] Mao Xiaoguang, Deng Yongjin, A General Model for Component-Based Software Reliability, Proceedings of the 29th EUROMICRO Conference "New Waves in System Architecture” (EUROMICRO’03) ,2003.

[7] SHERIF YACOUB, BOJAN CUKIC AND HANY H.AMMAR ,A Scenario-Based Reliability Analysis Approach for Component-Based Software ,IEEE,2004.

[8] Richard W. Selby, Enabling Reuse-Based Software Development of Large-Scale Systems, IEEE Computer Society, 2005.

[9] Yoshinobu Tamura and Shigeru Yamada, A Method of User-oriented Reliability Assessment for Open Source Software and Its Applications, IEEE, 2006.

[10] WANG Dong HUANG Ning YE Ming, Reliability Analysis of Component-Based Software Based on Relationships of Components , IEEE , 2008
[11] Fan Zhang, Xingshe Zhou, Junwen Chen, Yunwei Dong, A Novel Model for Component-based Software Reliability Analysis, IEEE, 2008.

[12] Francesca Saglietti, Florin Pinte, Sven Söhnlein, Integration and Reliability Testing for Component-Based Software Systems , 35th Euromicro Conference on Software Engineering and Advanced Applications,2009.

[13] Atef Mohamed and Mohammad Zulkernine, Failure Type-Aware Reliability Assessment with Component Failure Dependency, IEEE, 2010.

[14] Deepak Panwar, Pradeep Tomar, New Method to Find the Maximum Number of Faultsby Analyzing Reliability and Reusability in Component-Based Software , IEEE ,2011.

[15] Aditya Pratap Singh , Pradeep Tomar, A New Model for Reliability Estimation of Component-Based Software , IEEE,2012

[16] Pankaj Jalote, Brendan Murphy, Mario Garzia, Ben Errez,Measuring Reliability of Software Products ,Microsoft Corporation,2003

[17] ShraddhaShukla, Ms. Kirti Seth,Failure Dependent Reliability Analysis for Component Based Software Systems,IEEE,2012

[18] Deping Zhang, Shuai Wang and Wujie Zhou, Software Reliability Estimation Method Based on Markov Usage Models Using Importance Sampling ,IEEE Computer society ,2012

[19] Franz Brosch, Heiko Koziolek, Barbora Buhnova ,Ralf Reussner, Architecture-Based Reliability Prediction with the Palladio Component Model, IEEE Computer society, 2012

[20] Zhendong Wu ,Research on sensitivity of componentbased reliability ,IEEE Computer society ,2012

[21] Thanh-Trung Pham, Xavier Défago ,Reliability Prediction for Component-based Systems: Incorporating Error Propagation Analysis and Different Execution Models, IEEE Computer society ,2012

[22] Lyu, M. R. (Ed.), Handbook of Software Reliability Engineering, IEEE Computer Society Press, 1999. 